This is an electronic version of an article published in Patient Education \& Counseling 66 (3):263-269. The original publication is available at www.elsevier.com @ Elsevier

\title{
Accessing dental anxiety online support groups: An exploratory qualitative study of motives and experiences
}

\author{
Heather Buchanan \& Neil S. Coulson
}

Institute of Work, Health \& Organisations, University of Nottingham, Nottingham, UK.

Running title: Dental phobia and online support

\section{Address for correspondence:}

Dr Heather Buchanan

Institute of Work, Health \& Organisations

University of Nottingham

8 William Lee Buildings

University Boulevard, Nottingham, NG7 2RQ

E-mail: heather.buchanan@nottingham.ac.uk

Telephone: +44[0] 1158467520 


\section{ABSTRACT}

Objectives: In recent years, Internet access has grown markedly providing individuals with new opportunities for online information retrieval, psychological advice and support. The objectives of the present study were to explore the context through which dentally anxious individuals access an online support group and the nature of their online experiences.

Methods: An online questionnaire was completed by 143 individuals who accessed the Dental Fear Central online support group bulletin board. Qualitative analysis was conducted on the responses.

Results: Analysis revealed three emergent themes which reflected the motives and experiences of individuals: 'Searching for help', 'Sharing fears' and 'I feel empowered'.

Conclusion: This exploratory study suggests that for most individuals accessing this online support group was a positive and beneficial experience.

Practice Implications: Online support groups may represent a convenient and beneficial tool that may assist certain individuals to confront their debilitating anxiety/phobia and successfully receive dental care.

Keywords: computer-mediated communication, dental anxiety, Internet, phobia, social support, qualitative. 


\section{INTRODUCTION}

\subsection{Dental anxiety and phobia: Prevalence and consequences}

Despite advances in treatment techniques and local anaesthesia, one of the most common fears is that of dentistry ${ }^{1}$, with studies indicating that approximately $7-12 \%$ of individuals experience high dental anxiety or dental phobia $^{2-4}$. The literature suggests that dental anxiety and phobia may have a significant impact on patients' lives. Firstly, it often leads to avoidance of the stimuli (the dental context), which can have a deleterious effect on oral health ${ }^{5}$. In those instances where a patient is able to attend the dentist, then they may take longer to treat, and as oral health degenerates treatment can become more complex ${ }^{6,7}$. Secondly, dental anxiety and phobia can have a wide-ranging and profound impact on individuals' daily lives ${ }^{8}$. Patients report significant psychological and social consequences of their anxiety or phobia; shame and embarrassment are common experiences ${ }^{9}$ with research indicating that patients often report widespread negative social life effects ${ }^{2,10}$ and a threat to self-respect and well-being ${ }^{11,12}$.

\subsection{Support groups for individuals with dental anxiety/phobia}

Support groups can provide an opportunity for individuals to share their past experiences and fears about dental care in a supportive environment. Crawford, Hawker \& Lennon ${ }^{13}$ conducted a qualitative evaluation of a support group for dentally anxious adults who were avoiding dental care, with a dentist as group leader. The authors report that the group was successful in many ways, including the provision of support from group members, confidence building and ability to have treatment at an anxiety clinic. However, a number of weaknesses were acknowledged, including inconvenience experienced through attendance at meetings over lunchtime and dependence on the dentist as a group leader.

In recent times, there has been a marked increase in the number of individuals who are searching for health-related information, advice and support 
on the Internet ${ }^{14,15}$, including information related to dentistry ${ }^{16}$. A growing number of studies have described the reasons for, and benefits associated with, online support group participation ${ }^{14,17-20}$. Such groups are not restricted by the temporal, geographical and spatial limitations typically associated with face-toface groups ${ }^{21}$. This means that individuals can send and receive messages at any time of the day or night, 7 days a week ${ }^{22}$. Furthermore, with asynchronous communication (e.g. bulletin boards; mailing lists), the participant is able to carefully consider their message and develop it at their own pace before posting it to the group. Online support groups may also bring together a more heterogeneous mix of individuals offering diverse perspectives, experiences, opinions and sources of information than might otherwise be the case. Indeed, the number of participants within the online group can be unlimited and its membership worldwide.

\subsection{Computer-mediated communication}

There are many important differences which exist between computer-mediated communication and face-to-face communication and there has been much debate as to whether this leads to a more or less personal form of communication. According to Walther \& Burgoon ${ }^{23}$, the absence of social context cues limits the extent to which the individual can get to know the person with whom they are communicating, thereby leading to a less personal form of conversation. Furthermore, group participants may devote less attention to the other participants. Without the presence of others to restrain them, their interest may focus elsewhere and not on the group or its participants. However, even when group participants do become immersed in the conversation, there is a greater likelihood that they may "flame" or insult others, to place greater emphasis on themselves, and to equate each other's status. As a consequence, the extent to which intimacy and trust is developed is limited and only impersonal communication is likely. However, it has also been noted ${ }^{24}$ that these effects may 
be limited to the early stages of a computer-mediated relationship and these impersonal aspects may decline over time.

An additional consideration within the context of the online support group is the amount and rate of information exchange which may be taking place. According to Social Penetration Theory ${ }^{23}$ as the communication extends across a longer period of time it is likely to become more personal in nature. However, it is interesting to note that this type of communication might start out even more personal than face-to-face interaction. Individuals may be less concerned about the initial impression they are creating because of the anonymity conferred by the Internet. Such anonymity may facilitate self-disclosure and assist individuals in discussing sensitive issues more easily or to venture opinions with less fear of embarrassment or negative judgement than in face-to-face groups ${ }^{14,25,26}$. In the context of dental anxiety and phobia, these advantages may be particularly relevant and may offer some insight into why anxious or phobic patients are turning to the Internet for advice and support.

\subsection{Aims of the study}

Dental anxiety and phobia can have a profound impact on the lives of individuals. With the growth in Internet support groups, and their many potential advantages, dentally anxious or phobic individuals may increasingly be choosing to access these groups. However, we are not aware of any published research, which considers the specific reasons why individuals may be accessing dental anxiety or phobia support groups nor their experiences of accessing such groups. Moreover, little is known about how (or even if) they feel the group has helped them with their anxiety or phobia or whether there are any disadvantages associated with accessing the group. It has also been noted that research into dental anxiety and

phobia draws predominantly upon quantitative instruments, with a paucity of research employing in-depth qualitative methodologies, detailing the patient's 
own experience and perceptions of their dental anxiety or phobia ${ }^{11,12}$. Therefore, the primary aim of this study was to explore, using a qualitative methodology, the context through which dentally anxious individuals choose to access an online support group and their online experiences and to explore the extent to which online communication within the support group context is personal and meaningful to participants.

\section{METHODS}

\subsection{Participants}

Participants were 143 individuals who accessed the Dental Fear Central group and chose to participate in our online study during the 2 month data collection period. This existing non-commercial online support group was established to provide information and support to individuals who are affected by dental anxiety. Participants were 16 to 64 years of age (Mean age $=36.57$ years) with $76.1 \%$ being female. As part of the background information requested, participants were asked to complete a validated dental anxiety measure. The Modified Dental Anxiety Scale (MDAS) ${ }^{27}$ comprises 5 items based on the dental experience (e.g. "If you were about to have your tooth drilled, how would you feel?") and is rated on a 5-point scale ranging from 'Not anxious' to 'Extremely anxious'. Previous studies have demonstrated good internal reliability for the $M^{27-28}$. The MDAS indicated that the sample were dentally anxious (mean = 20.03; SD=5.02) with $70 \%$ scoring above 19 (indicating a strong likelihood of dental phobia $\left.{ }^{27}\right)$.

In terms of country of residence, $51.7 \%$ were from the United States, $35.6 \%$ from the United Kingdom, $7.7 \%$ from Canada, 3.6\% from Australia, $0.7 \%$ from Germany and $0.7 \%$ from Slovenia. In regard to marital status, $28 \%$ were single, $49.7 \%$ were married, $16.1 \%$ were living with a partner, $2.1 \%$ were separated or divorced and $4.1 \%$ reported being in a relationship but did not live together. 


\subsection{Procedure}

Initial contact was established between the authors and the group administrator. The purpose of the study and methodology were explained to the administrator and comments on the proposed structure and content of the online questionnaire invited. In addition, it was explained that approval for the study had been obtained from the Psychology subject group ethics committee from the lead author's institution (ethical approval code 009/04). Approval was then obtained from the administrator and a 'news item', as well as a message posted to the bulletin board by the administrator, invited group members to participate in the study. Individuals who did wish to participate were invited to visit a webpage which provided further information concerning the nature of the study and their rights as a participant in the research process. Each participant was asked to provide a 6-digit password through which their data could be identified should they wish, at any point, for it to be withdrawn from the study (no participant chose to take this option). To prevent individuals making multiple responses the data collection software was set to recognise and prevent responses originating from the same IP address. Additionally, all participants were asked if their responses could be quoted in any dissemination. Contact details for both authors (which included an email address) were also provided to each participant prior to and after electronic submission of their responses.

Participants were invited to respond to a series of open-ended questions designed to explore their experiences of accessing the online group (see Box 1).

Similar questions and methodology have previously been employed in the examination of online support groups (e.g. Coulson \& Knibb, in press) ${ }^{29}$ and were agreed by the administrator of the Dental Fear Central support group.

\section{$<$ insert box 1 about here >>}




\subsection{Data analysis}

The responses to questions provided by participants were analysed using phenomenological thematic analysis according to the guidelines as set out by Colaizzi $^{30}$ and Osborne ${ }^{31}$. The purpose of this analysis is to ask 'What is this kind of experience like?' Phenomenological research strives for a richer understanding of, and insight into, the lived experiences of individuals. In the present study, the 'lived' experience is an online experience. It is important to note that a phenomenological approach endeavours to describe and interpret meanings. It does not aim to quantify the frequency of certain behaviours or viewpoints but rather it focuses upon the relationship between the individual and the lived world that the individual inhabits.

In the context of the present study, the analysis sought to understand the meanings and significance which individuals attached to their online experience in the context of dental anxiety and phobia. In order to do this, our analysis looked across both questions and respondents in order to elicit broad themes to aid our understanding of respondents' experiences. Both authors read all of the participant responses in full, several times, to ensure familiarity with the data. Notes were then taken and recorded on the transcripts to reflect initial impressions. Further systematic reading of the transcripts, and the notes made, enabled the identification of possible conceptual themes that captured the initial notes. There then followed discussion of the identified themes by both authors, and these were solidified into three common themes.

\section{Results}

The results of the analysis revealed 3 emergent themes related to the context and circumstances through which individuals accessed the group and their experiences of using the online support group.

'Searching for help'- In many instances, respondents appeared to acknowledge a specific dental problem, which required treatment (e.g. bleeding 
gums, broken or decayed teeth). However, in accepting the need for treatment, participants also recognised their fears concerning dentists and dental procedures as an overwhelming barrier. Faced with this seemingly insurmountable challenge, and often too ashamed to discuss their 'irrational' fears with family and friends, individuals sought information, advice and support from the Internet. It was typically through surfing and searching the world-wide-web that respondents often first found out about online support groups such as Dental Fear Central. As one respondent explains "I began reading online support groups when I started considering returning to the dentist. My wisdom teeth were decaying and clearly needed to come out - one of them was falling out in chunks. I was completely terrified and didn't know what to do, so I started doing a bit of research on wisdom teeth on the internet. At that point I didn't really know that such a thing as 'dental phobia' as a proper condition existed, but I learned that as I was researching. It was through googling 'dental phobia' that I found some online support groups" (28 year old, female).

'Sharing fears'- For almost all respondents there appeared to be great comfort derived from the knowledge that they were not alone. In many cases, individuals described how they were unaware of anyone in their everyday social network that shared similar fears and anxieties. As a result, feelings of isolation were common and the burden of this 'totally irrational fear' weighed heavy on the minds of respondents, "It is nice to know that I am quite normal in my anxieties and phobias" (50 year old, female). However, the online support group appeared to offer some hope and through reading the messages posted by others, individuals often realised that their experiences were not unique or uncommon. For many, this realisation was instrumental in helping them challenge the fears that had held them back for so long, as one member describes; "Well I can say I clearly do not feel alone, and actually did find the name of a dentist who is 2 hours from me, so I might get the courage to call him up and see what he can 
do" (32 year old, female). This also extended to those members who had read messages but had not actually posted themselves, "I am a new member and have yet to post in the group...however just reading what others have posted has already given me some peace of mind. I know I am not alone in this" (24 year old, female).

Many respondents described their feelings at being able to share their dental experiences and stories with others. For many, the ability to discuss their past dental history was a novel and cathartic experience. Whilst some respondents still reported feeling anxious or ashamed to share their fear, the particular mode of communication required to participate in the group appeared helpful. By communicating online, individuals were conferred a degree of anonymity and control which would not have been possible in a traditional faceto-face exchange. For some, the benefits of this 'virtual' anonymity were profound and through observing the patterns of disclosure and reciprocal disclosure, which was common place within the group, those individuals who wished to share their experiences were eventually able to do so. As one individual notes "It's anonymous and feels like a safe environment which is non-judgmental as everyone has experienced [or is still experiencing] fear or anxiety at some level" (26 year old, female). Of particular benefit to individuals was the empathy and understanding, which was offered from others who truly understood their fears and anxieties. "Being able to find other caring and sympathetic people who know how you feel. Most people I talk to all say the same thing 'oh nobody likes going to the dentist'. They don't understand that this isn't just a little nervousness, it is full blown fear. This support group has been a good place to come anytime I need reassurance" (26 year old, female). It was often the case that respondents specifically compared the responses and reactions of group members with non-group members "....it was a place where I could post questions without being told that I am stupid and make a fuss over nothing (like my childhood dentists and others since would say)" (46 year old, female). 
Through the messages posted to the online group, individuals were able to obtain both practical information, such as dentist recommendations, and a rich and varied source of information about the realities of coping with dental anxiety. As one participant commented "They helped me to talk about things and also gave some useful advice about finding a dentist and eventually communicating my fears to them" (32 year old, female). In particular, participants welcomed the detailed discussion about dental treatments and practices and were able to share and discuss potential coping strategies, not only in the dental context (e.g. visiting the dentist or having work done) but also in daily life (e.g. coping with broken or discoloured teeth; feelings of stigma and shame). For many, the benefit, which was derived, came from reading other members' positive experiences about coping. It was commonplace for individuals to post updates, for example, following a dental appointment. These were warmly welcomed by individuals and as one participant noted: "There are some incredible success stories in the forum that have already been encouraging for me. When someone posts about what they are going to have done and how scared they are, they sound exactly like me. Then when they get through that appointment they were so dreading and fearing, they post again and say how well it went and that it wasn't as bad as they thought. I figure if they can do it, maybe I can too" (26 year old, female).

'I feel empowered'- Through accessing the messages posted by others facing similar challenges and the guidance, support and encouragement offered, individuals appeared empowered to begin their own journey towards confronting their fear. The starting point for each journey was often varied and the path different, but common to all was a sense that the online group has provided them with the impetus to try and conquer their fears and to move forward. One participant reports how it helped her to both make a dental appointment and then go through with it. "It gave me the courage to finally make my appointment. And 
the words of encouragement helped me to stay at the office and not run for my life" (40 year old, female). Participants often discussed their empowerment in terms of a growth in confidence about communicating their fears to the dental team. "Knowing that I am not the only one who is fearful has made me feel more confident. (If I had to find another dentist, I would feel more confident about saying that I am fearful and also saying what is helpful). Also, I am now much better informed about dental treatments, which again has made me feel more confident" (46 year old, female). For some the group helped them work out why they were fearful and the specific target of their anxiety, as well as the strength to speak out. "It has helped me define and face what about the dental clinic setting that causes my fears. I have also learned to have enough courage to speak for myself and to be my own advocate" (47 year old, male).

\section{Discussion}

\subsection{Discussion}

To date, there has been relatively little attention given to understanding the online experiences of dentally anxious or phobic patients. The aims of the present study, therefore, were to investigate the context and circumstances which led dentally anxious individuals to access an online support group and their experiences of using the group. We found that employing phenomenological thematic analysis within this study provided a rich understanding of individuals' experiences of the online group and its significance and role within their lives. The emergent themes suggest that feeling less isolated, sharing experiences with others, giving and receiving information and advice and becoming empowered are key functions, important consequences and meaningful experiences for the group.

For most group members the initial prompt for seeking information, advice and support from the Internet was a specific dental problem (e.g., bleeding gums) that required treatment but which they had felt unable to obtain as a 
result of their fears. It has been well documented that dental anxiety and phobia can have a serious negative impact on oral health, as individuals tend to avoid accessing dental care for extended periods of time ${ }^{5}$. Locating and accessing the group appeared to have an immediate and clear benefit for individuals in that they no longer felt that they were alone in trying to cope with their dental anxiety or phobia but rather were now part of a larger community. The online group appeared to offer individuals a virtual meeting place through which they could communicate with others who have the same or similar problems.

At the heart of the online experience for group members was the opportunity to share thoughts, feelings, opinions, experiences and stories about their dental fear within a safe and supportive environment. Furthermore, the online support group appeared to provide great comfort to members as they could read and exchange messages on a range of topics without the worry that others would not understand their fear, or that they would not be taken seriously. Previous research suggests that support seeking is highest for conditions that are stigmatising or embarrassing ${ }^{32}$, which is reflective of how the group members describe their phobia. Our findings suggest that the benefits of online support groups can be important for those who consider themselves to be isolated through fear, stigma or embarrassment.

Whilst there are evidently some similarities between online and traditional face-to-face dental anxiety and phobia support groups (such as sharing opinions and experiences ${ }^{13}$ ), it is arguably the computer-mediated asynchronous nature of the communication (i.e. bulletin board), which offered additional and unique benefits to this group. The format and structure of the online bulletin board clearly benefited those who were accessing the group. Typically, an individual would start a discussion topic (or thread) by posting a question or comment/story to which others were able to reply. Subsequent replies would be organised and indented under the message to which they related and listed in the order in which they were posted. As a result, those accessing the group would be able to read 
messages in sequence which were organised and archived according to topic. Through this system of threading discussion topics, those accessing the group can search for a range of specific topics and can read messages at their leisure. Other benefits resulted from the anonymity which this mode of communication offered to those who wished to post messages. Participants were able to control the amount of personal information which they disclosed to the group and this appeared to reassure those who were initially anxious at revealing their very personal fears and anxieties. This finding is mirrored in other studies ${ }^{17,22}$ and suggests that anonymity is an important benefit derived from the computermediated nature of the communication which takes place.

Through the support group, members were able to obtain rich and varied sources of emotional and informational support, which focused on the everyday realities of coping with dental anxiety or phobia. This advice included coping with the oral health implications (e.g., decaying teeth) and the psychosocial consequences of their fear (stigma, shame and embarrassment). Informational support often took the form of providing members with practical information, such as new treatment advances and contact details of supportive dentists. These findings are consistent with other studies which have suggested that emotional and informational support are often the most frequent types of social support given within online support groups ${ }^{17,33,34}$.

By accessing the group, some individuals appear to be empowered to begin their journey towards conquering their dental anxiety or phobia. Of particular benefit appeared to be the many success stories which were shared between members and these messages served as reminders that dental anxiety or phobia can be overcome. Whilst some individuals suggested that the online support group had been instrumental in overcoming their anxiety or phobia, future research should aim to test out this assertion using a robust prospective study design. Indeed, in reviewing the efficacy of online support groups, the current state of the literature and its findings is mixed ${ }^{35}$. However, our findings do 
support the view that communication within such online groups can indeed be highly personal as evidenced by the detailed accounts of dental anxiety and the sharing of fears and concerns which participants themselves stated were irrational and embarrassing.

Within our study, the majority of participants were female. Whilst it may be the case that females were simply more motivated to take part in our study, past research has suggested that females are more likely than males to use the Internet for health information ${ }^{36}$ and access some online support groups ${ }^{37}$. Nevertheless, our findings did not suggest any discernible differences in either the reasons given for accessing this online group nor its advantages in terms of coping with the challenges of dental anxiety. However, future research should seek to explore the issue of gender differences more fully as other work (e.g. Sullivan, $2003^{38}$ ) does suggest that females can differ from males in the nature and type of support they provide each other within online groups. Similarly, there exists a dearth of literature which explores the relationship between sociodemographic characteristics and the experiences of individuals online. Since the present study aimed to look for patterns and themes across these groupings we are limited in the commentary that can be provided but future research may seek to examine key socio-demographic variables within the context of the online support group use as well as computer-mediated communication.

There are a number of methodological issues which are raised by this study and should be considered. Firstly, the participants may not be representative of all dentally anxious and phobic individuals who use Internet support groups as the invitation to participate in our study was posted on only one online support group bulletin board. It is conceivable that other support groups have their own unique aims, goals and dynamics, which may differ from those described in the present study. Secondly, those participants who chose to complete our online questionnaire may not be representative of those who access this or similar online support groups. It may be the case that only those who held 
a particularly positive view of this group chose to participate. Some individuals may have accessed the group but derived little benefit (or even negatively evaluated the group) and therefore either chose not to participate in the study or had ceased to access the support group prior to the study being conducted. A final consideration relates to the extent to which our respondents actually participate in the online support group. Our focus was on exploring the views of those who had accessed the group but we did not limit ourselves to those who had actually posted or replied to a message. Since messages posted to the bulletin board were public and did not require registration with the group to read them many people could potentially access the group without actually participating. Indeed, within the present study several respondents indicated that they had not yet posted a message but nevertheless had benefited from reading those posted by other members. Future research may usefully compare active members with those who 'lurk' (i.e. those who read messages but who do not themselves post a message) as recent evidence suggests ${ }^{39}$ that although 'lurkers' do seek answers to questions, their overall satisfaction with the community experience may be lower than those who actively participate.

\subsection{Conclusion}

The present study offers a unique insight into the context and circumstances which led dentally anxious individuals to access an online support group and their experiences of using the group. The researchers were able to explore the online experiences of a sizeable, diverse and international group of individuals. As dental anxiety and phobia can have a significant daily impact on individuals' lives, these findings are encouraging and suggest that online support groups may benefit certain individuals. Future research, however, should employ a prospective randomised control design in order to monitor anxiety across time for those individuals accessing the group for the first time. 


\subsection{Practice implications}

This methodology represents a novel and potentially important route through which dental staff and psychologists can understand more fully the challenges facing those who are dentally anxious or phobic. Moreover, online support groups may represent a new, accessible, convenient and beneficial method through which dentists and patients can communicate to help individuals overcome this debilitating anxiety/phobia and successfully receive dental care.

\section{Acknowledgements}

The authors would like to thank the administrator and participants of the Dental Fear Central discussion board for consenting to take part in this study and allowing us to use quotes from their responses.

\section{References}




\section{References}

[1] Kent G. Dental phobias. In: Davey GCL, ed. Phobias: a handbook of theory, research and treatment. New York: Wiley \& Sons, 1997.

[2] Locker D. Psychosocial consequences of dental fear and anxiety. Community Dent Oral Epidemiol 2003; 31: 144-151.

[3] Sohn W, Ismail AI. Regular dental visits and dental anxiety in an adult dentate population. J Am Dent Assoc 2005; 136: 58-66.

[4] Vassend O. Anxiety, pain and discomfort associated with dental treatment. Behav Res Ther 2003; 31: 659-66.

[5] Schuller AA, Willumsen T, Holst D. Are there differences in oral health and oral health behavior between individuals with high and low dental fear? Community Dent Oral Epidemiol 2003; 31: 116-121.

[6] Corah NL, O'Shea RM, Skeels DK. Dentists' perceptions of problem behaviours in patients. J Am Dent Assoc; 104; 829-33.

[7] Skaret E, Raadal M, Kvale G, Berg E. Factors related to missed and cancelled dental appointments among adolescents in Norway. Eur J Oral Sci 2000; 108: 175-83.

[8] Cohen SM, Fiske J, Newton JT. The Impact of Dental Anxiety on Daily Living. Br Dent J 2000; 189: 385-90.

[9] Moore R, Brødsgaard I, Rosenberg N. The contribution of embarrassment to phobic dental anxiety: a qualitative research study. BMC Psychiatry 2004; 4: 10.

[10] Berggren U. Psychosocial effects associated with dental fear in adult dental patients with avoidance behaviours. Psychol Health 1993; 8: 185-96.

[11] Abrahamsson KH, Berggren U, Hallberg L, Carlsson S. Ambivalence in Coping with Dental Fear and Avoidance: A Qualitative Study. J Health Psychol 2002a; 76: 653-664.

[12] Abrahamsson KH, Berggren U, Hallberg L, Carlsson S. Dental phobic patients' view of dental anxiety and experiences in dental care: A qualitative study. Scand J Caring Sci 2002b;16: 188-96.

[13] Crawford AN, Hawker BJ, Lennon MA. A dental support group for anxious patients. Br Dent J 1997; 183: 57-62.

[14] Madara EJ. (1997). The mutual-aid self-help online revolution. Soc Policy 1997; Spring; 20-26. 


\section{Box 1. Questions posed to participants}

In your own words, could you tell us why you decided to participate in an online support group?

In your view, has being a member of an online support group made any difference to how you cope with your dental anxiety/phobia? If so, could you give some examples?

What do you consider to be the main advantages of participating in an online support group?

Are there any problems you have experienced in participating in an online support group? 